Pacific Journal of Mathematics

INDEX FOR PAIRS OF FINITE NON NEWMAN ALGEBRAS 


\title{
INDEX FOR PAIRS OF FINITE VON NEUMANN ALGEBRAS
}

\author{
PAUL Jolissaint
}

\begin{abstract}
The Jones' index of a pair $N \subset M$ of finite von Neumann algebras with finite dimensional centers has been given two definitions: one ring-theoretic, and one using Markov traces. We extend here the second definition to the case of finite, $\sigma$-finite von Neumann algebras and we show that the two definitions agree when the algebras are direct sums of finite factors. We also study Markov traces on such pairs.
\end{abstract}

Introduction. The purpose of the present article is twofold:

(1) If $N \subset M$ is a pair of direct sums of finite factors, we prove that the index $[M: N]$ defined in Chapter 3 of [3] is equal to the ring-theoretic index introduced in Chapter 2 of [3].

(2) We give a definition of the index for a pair as above in terms of canonical objects associated to $N$ and $M$ such as center-valued traces and coupling operators.

In fact, we present a solution of problem (2) providing a framework in which problem (1) is easily solved. More precisely, let $M$ be a finite, $\sigma$-finite von Neumann algebra and let $N$ be a von Neumann subalgebra of $M$ containing the identity of $M$. If $N$ is of finite index in $M$, i.e. if $M$ acts on some Hilbert space $H$ in such a way that the commutant $N_{H}^{\prime}$ of $N$ is finite, and that the coupling operators $c_{M}(H)^{ \pm 1}$ and $c_{N}(H)^{ \pm 1}$ are bounded, then we define two bounded, linear, normal maps

$$
C_{N}^{M} \in L_{*}(Z(N)) \text { and } D_{N}^{M} \in L_{*}(Z(M))
$$

which do not depend on the chosen representation and which have the same spectral radius.

Thus the index of $N$ in $M$, denoted by $[M: N]$, is the common spectral radius of $C_{N}^{M}$ and $D_{N}^{M}$.

Let $\left(M, L^{2}(M), J, P\right)$ be the standard form of $M([4])$. The basic construction associated to the pair $N \subset M$ gives the finite von Neumann algebra $J N^{\prime} J$, denoted by $\left\langle M, e_{N}\right\rangle$. Then $M$ is of finite index in $\left\langle M, e_{N}\right\rangle$ and $\left[\left\langle M, e_{N}\right\rangle: M\right]=[M: N]$, so it is possible to 
iterate the basic construction and we get a tower of finite von Neumann algebras $\left(M_{k}\right)_{k \geq 0}$ with

$$
M_{0}=N \subset M_{1}=M \subset \cdots \subset M_{k} \subset M_{k+1} \subset \cdots
$$

where $M_{k+1}$ comes from the basic construction associated to the pair $M_{k-1} \subset M_{k}$. Let us state the main result of this paper:

Theorem I. Assume that $N$ and $M$ are finite or countable direct sums of finite factors, $N$ being of finite index in $M$. Then the tower $\left(M_{k}\right)$ is isomorphic to the ring-theoretic tower of Chapter 2 of [3], and

$$
[M: N]=\underset{k \rightarrow \infty}{\limsup }\left[\operatorname{rk}\left(M_{k} \mid M_{0}\right)\right]^{1 / k}
$$

where $\operatorname{rk}\left(M_{k} \mid M_{0}\right)$ denotes the smallest possible number of generators of $M_{k}$ as a right $M_{0}$-module.

Except for a technical lemma concerning some suitable trace on $M$, Theorem I follows readily from the following proposition which gives a nice relationship between the endomorphisms $C_{N}^{M}$ and $C_{N}^{M_{k}}$ :

Proposition II. Suppose that $N \subset M$ is a pair of finite, $\sigma$-finite von Neumann algebras, $N$ being of finite index in $M$. Then we have for every positive integer $k$ :

$$
C_{N}^{M_{k}}=\left(C_{N}^{M}\right)^{k}
$$

We prove similarly that $D_{N}^{M_{k}}=\left(D_{M_{k-1}}^{M_{k}}\right)^{k}$.

The motivation for the choice of the map $D_{N}^{M}$ is explained by the following result:

Proposition III. (1) Let $N \subset M$ be a pair as in Proposition II and let $\varphi$ be a normal, faithful, finite trace on $M$. Then $\varphi$ is a Markov trace of modulus $\beta$ for the pair $N \subset M$ (see Definition 5.1) if and only if:

$$
\varphi \circ D_{N}^{M}=\beta \varphi \mid Z(M) .
$$

(2) Suppose that $\varphi$ is a Markov trace of modulus $\beta$ for $N \subset M$. Then its extension to $\left\langle M, e_{N}\right\rangle$ is a Markov trace of modulus $\beta$ for $M \subset\left\langle M, e_{N}\right\rangle$.

We will see that Markov traces do not always exist, but classical Perron-Frobenius theory implies that such traces exist when $Z(M)$ 
and $Z(N)$ are finite dimensional and when $Z(N) \cap Z(M)=\mathbb{C}$ (see [3], 3.7.4). When the pair $N \subset M$ has a Markov trace $\operatorname{tr}_{M}$ of modulus $[M: N]$, then the triple $\left(N, M, \operatorname{tr}_{M}\right)$ behaves more or less like a pair of factors, as the next two results show:

THEOREM IV. If the pair $N \subset M$ admits a Markov trace $\operatorname{tr}_{M}$ of modulus $[M: N]$ then the basic construction $\left\langle M_{k}, e_{N}\right\rangle$ associated to the pair $N \subset M_{k}$ is isomorphic to $M_{2 k}$ for every $k$.

Remark that Theorem IV is a generalization of Theorem 2.6 of [7].

Finally, the last proposition may be compared to the definition of index in the case of factors [5]:

Proposition V. Assume that $\operatorname{tr}_{M}$ is a normalized Markov trace of modulus $[M: N]$ for the pair $N \subset M$. Denote by $\operatorname{tr}_{N}$ the restriction to $N$ of $\operatorname{tr}_{M}$. Then the pair $M^{\prime} \subset N^{\prime} \subset B\left(L^{2}(M)\right)$ admits a normalized Markov trace $\operatorname{tr}_{N^{\prime}}$ of modulus $\left[N^{\prime}: M^{\prime}\right]=[M: N]$ and we have for every $\xi$ in $L^{2}(M)$ :

$$
[M: N] \operatorname{tr}_{N^{\prime}}\left(e_{\xi}^{(N)}\right)=\operatorname{tr}_{N}\left(e_{\xi}^{\left(N^{\prime}\right)}\right),
$$

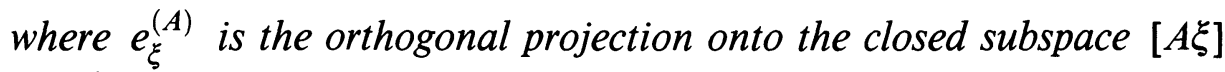
of $L^{2}(M)$.

I would like to thank P. de la Harpe, V. Jones and G. Skandalis for their suggestions and comments during the preparation of this article.

1. Preliminaries. Throughout this paper $M$ will be a finite, $\sigma$-finite von Neumann algebra, i.e. $M$ admits a normal, faithful finite trace. $Z(M)$ denotes the center of $M$ and $\hbar_{M}$ its canonical $Z(M)$-valued trace. If $M$ acts on some Hilbert space $H$ in such a way that its commutant $M_{H}^{\prime}$ is finite, then there exists a unique positive, selfadjoint operator $c_{M}(H)$, which is affiliated with $Z(M)$ and invertible in the sense of unbounded operators, such that

$$
\left(e_{\xi}^{\left(M^{\prime}\right)}\right)^{\natural_{M}}=c_{M}(H)\left(e_{\xi}^{(M)}\right)^{\natural_{H}^{\prime}},
$$

for every $\xi$ in $H$, where $e_{\xi}^{(A)}$ is the projection onto the closed subspace $[A \xi]$ of $H$ and where $t_{H}^{\prime}$ denotes the trace on $M_{H}^{\prime}$. The operator $c_{M}(H)$ is called the coupling operator associated to $M$ and $H$ and it possesses the following properties:

(i) $c_{M^{\prime}}(H)=c_{M}(H)^{-1}$; 
(ii) $c_{q M}(q H)=q c_{M}(H)$ for every central projection $q$;

(iii) if $e^{\prime}$ is a projection in $M_{H}^{\prime}$ with central support 1, then:

$$
c_{M_{e^{\prime}}}\left(e^{\prime} H\right)=e^{\prime}\left(e^{\prime}\right)^{\natural_{H}^{\prime}} c_{M}(H) .
$$

Let us recall that if $e^{\prime}$ is as in (iii) above, one has:

$$
\left(x_{e^{\prime}}\right)^{\natural_{e^{\prime}}}=e^{\prime}\left(x^{\natural_{M}}\right) \text { and } \quad\left(x_{e^{\prime}}^{\prime}\right)^{\natural_{e^{\prime}}^{\prime}}=e^{\prime}\left(e^{\prime t_{H}^{\prime}}\right)^{-1}\left(e^{\prime} x^{\prime} e^{\prime}\right)^{\natural_{H}^{\prime}},
$$

for $x$ in $M$ and $x^{\prime}$ in $M_{H}^{\prime}$, where $\natural_{H}^{\prime}$ (resp. $\natural_{e^{\prime}}, \natural_{e^{\prime}}^{\prime}$ ) denotes the canonical trace on $M_{H}^{\prime}$ (resp. $\left.M_{e^{\prime}}, M_{e^{\prime}}^{\prime}\right)$. (See [2] or [8]).

Two projections $e$ and $f$ of $M$ are said to be equivalent if there exists $u$ in $M$ such that: $u^{*} u=e$ and $u u^{*}=f$. We write this fact: $e \sim f$. If a projection $e$ is equivalent to a subprojection of $f$, we write: $e \precsim f$. By Corollary V.2.8 of [10], if $e$ and $f$ are projections in $M$, then $e \precsim f$ if and only if $e^{\natural_{M}} \leq f^{\natural_{M}}$.

Let $\left(M, L^{2}(M), J, P\right)$ be the standard form of $M([4]): M$ acts normally and faithfully on the Hilbert space $L^{2}(M), P$ is a selfdual cone of $L^{2}(M), J$ is an antilinear involution on $L^{2}(M)$ and the following relations hold:

(1) $J M J=M^{\prime}$;

(2) $J c J=c^{*}$ for $c$ in $Z(M)$;

(3) $J \xi=\xi$ for $\xi$ in $P$;

(4) $\operatorname{aJaJ}(P)$ is contained in $P$ for $a$ in $M$.

$M_{*}$ denotes the predual of $M$ and $M_{*, c}$ denotes the set of central elements of $M_{*}$, i.e. those elements $\varphi$ in $M_{*}$ such that $\varphi(x y)=$ $\varphi(y x)$ for every $x, y$ in $M$. A normal, finite trace on $M$ is then a positive element of $M_{*, c}$. The preadjoint of the canonical trace $\natural_{M}$ is an isometric isomorphism from $Z(M)_{*}$ onto $M_{*, c}$ whose inverse is the map $\varphi \mapsto \varphi \mid Z(M)$ from $M_{*, c}$ onto $Z(M)_{*}$, i.e.

$$
(\varphi \mid Z(M))\left(x^{\natural_{M}}\right)=\varphi(x) \text { for } x \text { in } M \text { and } \varphi \text { in } M_{*, c} \text {. }
$$

If $F$ is a bounded, normal linear map from $Z(M)$ to the center $Z(N)$, where $N$ is some finite, $\sigma$-finite von Neumann algebra, we denote by $F_{*}$ the map from $N_{*, c}$ to $M_{*, c}$ defined by

$$
F_{*}(\varphi)=\varphi \circ F \circ \natural_{M}
$$

for every $\varphi$ in $N_{*, c}$. 
We end this section with two lemmas which are certainly well known, but we could not find any reference for them:

LEMMA 1.1. Suppose that $M$ is contained in $B(H)$ and let $e$ be a projection in $M$ such that there exist $\xi_{1}, \ldots, \xi_{n}$ in $H$ with

$$
e=\bigvee_{i=1}^{n} e_{\xi_{i}}^{\left(M^{\prime}\right)}
$$

Then there exist $\eta_{1}, \ldots, \eta_{n}$ in $H$ such that the projections $e_{\eta_{t}}^{\left(M^{\prime}\right)}$ are pairwise orthogonal and

$$
e=\sum_{i=1}^{n} e_{\eta_{i}}^{\left(M^{\prime}\right)}
$$

Proof. Set $e_{\xi}=e_{\xi}^{\left(M^{\prime}\right)}$ for $\xi$ in $H$. We prove the lemma by induction on $n$ : the assertion is obvious for $n=1$; assume that it is true for some $n$, and let $e$ be a projection in $M$ such that

$$
e=\bigvee_{i=1}^{n+1} e_{\xi_{i}}
$$

with $\xi_{1}, \ldots, \xi_{n+1}$ in $H$. Set

$$
f=\bigvee_{i=2}^{n+1} e_{\xi_{i}}
$$

There exist $\eta_{2}, \ldots, \eta_{n+1}$ in $H$ such that the $e_{\eta_{t}}$ are pairwise orthogonal with sum $f$. Moreover

$$
e-f=e_{\xi_{1}} \vee f-f \sim e_{\xi_{1}}-e_{\xi_{1}} \wedge f \leq e_{\xi_{1}},
$$

and hence there exists $\eta_{1}$ in $H$ with $e-f=e_{\eta_{1}}$.

LEMMA 1.2. Let $N$ be a von Neumann subalgebra of $M$ containing the identity of $M$. Then:

(1) $\left(y^{\natural_{N}}\right)^{\natural_{M}}=y^{\natural_{M}}$ for every $y$ in $N$;

(2) if $y \in N_{+}$is such that $y^{\natural_{N}}$ is invertible, then $y^{\natural_{M}}$ is invertible.

Proof. (1) We have to prove that one has for every $\zeta$ in $Z(M)_{*}$ :

$$
\zeta\left(\left(y^{\natural_{N}}\right)^{\natural_{M}}\right)=\zeta\left(y^{\natural_{M}}\right) \text {. }
$$

Fix some $\zeta$ in $Z(M)_{*}$ and let $\varphi_{\zeta}$ be the unique element of $M_{*, c}$ such that $\varphi_{\zeta} \mid Z(M)=\zeta$. Then $\varphi_{\zeta}\left(y^{\natural_{N}}\right)=\varphi_{\zeta}(y)$ since $y^{\natural_{N}}$ belongs to 
the norm-closed convex hull of the set $\left\{v y v^{*} ; v\right.$ unitary in $\left.N\right\}$ and since $\varphi_{\zeta} \mid N$ is central. Hence

$$
\zeta\left(\left(y^{\natural_{N}}\right)^{\natural_{M}}\right)=\varphi_{\zeta}\left(y^{\natural_{N}}\right)=\varphi_{\zeta}(y)=\zeta\left(y^{\natural_{M}}\right) \text {. }
$$

(2) There exists a positive number $c$ such that $y^{\natural_{N}} \geq c$. One gets from (1): $y^{\natural_{M}}=\left(y^{\natural_{N}}\right)^{\natural_{M}} \geq c$, and $y^{\natural_{M}}$ is invertible.

2. Finite representations; index. In this section, $M$ denotes a finite, $\sigma$-finite von Neumann algebra and $N$ is a von Neumann subalgebra of $M$ containing the identity of $M$.

DEFINITION 2.1. (1) A (normal, faithful) representation $(\pi, H)$ of $M$ is a finite representation of $M$ if the commutant $\pi(M)^{\prime}$ of $\pi(M)$ is finite and if the coupling operators $c_{\pi(M)}(H)$ and $c_{\pi(M)^{\prime}}(H)$ are bounded operators.

(2) A representation $(\pi, H)$ of $M$ is a finite representation of the pair $N \subset M$ if it is a finite representation for $M$ and $N$.

Notation. Let $(\pi, H)$ be a representation of $M$. We denote by $M_{H}^{\prime}$ (resp. $\left.N_{H}^{\prime}\right)$ the commutant of $\pi(M)$ (resp. $\pi(N)$ ) in $B(H)$. We simply denote by $M^{\prime}$ (resp. $N^{\prime}$ ) the commutant of $M$ (resp. $N$ ) in $B\left(L^{2}(M)\right)$.

LemMA 2.2. Suppose that $M$ is contained in $B(H)$ and that the action of $M$ on $H$ is a finite representation of $M$. Let $(\pi, K)$ be another finite representation of $M$. Then there exist:

(1) an integer $n \geq 1$.

(2) a projection $e^{\prime}$ in $M_{H}^{\prime} \otimes B\left(K_{n}\right)$ with invertible trace $\left(K_{n}\right.$ denotes the Hilbert space of dimension $n)$,

(3) a surjective isometry $u$ from $K$ onto $e^{\prime}\left(H \otimes K_{n}\right)$, such that

$$
\pi(x)=u^{*}\left(x \otimes 1_{n}\right) e^{\prime} u \text { for } x \text { in } M .
$$

Proof. Let $c_{M}(K)$ be the element of $Z(M) \subset B(H)$ such that $\pi\left(c_{M}(K)\right)=c_{\pi(M)}(K)$. There exists an integer $m \geq 1$ such that $c_{M}(K) \leq m$ and $c_{M}(H) \geq m^{-1}$. By Propositions 3 and 6, pages 300 and 302, of [2], and by Lemma 1.1, the identity of $M_{K}^{\prime}$ is the orthogonal sum of $m$ cyclic projections, and every positive normal form on $M$ is the sum of $m$ cyclic forms. Set $n=m^{2}$. By Theorem 3 , page 61 , of [2], there exists a projection $e^{\prime}$ in $M_{H}^{\prime} \otimes B\left(K_{n}\right)$ such that $\pi(M)$ is spatially isomorphic to $\left(M \otimes 1_{n}\right) e^{\prime}$. Moreover

$$
c_{M}(K) \otimes 1_{n}=n\left(e^{\prime}\right)^{\natural_{n}^{\prime}} c_{M}(H) \otimes 1_{n}
$$


which shows that $\left(e^{\prime}\right)^{\natural_{n}^{\prime}}$ is invertible as $c_{M}(H)$ and $c_{M}(K)$ are. (We denote by $\mathfrak{a}_{n}^{\prime}$ the canonical trace on $M_{H}^{\prime} \otimes B\left(K_{n}\right)$.)

We are presenting now the main tools needed in this paper:

THEOREM 2.3. Let $N \subset M$ be a pair as above.

(a) The following conditions are equivalent:

(a1) there exists a finite representation of the pair $N \subset M$;

(a2) the standard representation of $M$ is a finite representation of the pair $N \subset M$;

(a3) any finite representation of $M$ is a finite representation of the pair $N \subset M$.

(b) Assume that one of the conditions in (a) holds, and let $H$ be a finite representation of the pair $N \subset M$. Define $S_{N}^{M}: Z(M) \rightarrow Z(N)$ and $T_{N}^{M}: Z(N) \rightarrow Z(M)$ by

$$
S_{N}^{M}(z)=c_{N}(H)\left(c_{M}(H)^{-1} z\right)^{\natural_{N}^{\prime}}
$$

and

$$
T_{N}^{M}(w)=w^{\natural_{M}}
$$

for every $z$ in $Z(M)$ and every $w$ in $Z(N)$. Set also

$$
C_{N}^{M}=S_{N}^{M} T_{N}^{M} \quad \text { and } \quad D_{N}^{M}=T_{N}^{M} S_{N}^{M}
$$

(b1) The maps $S_{N}^{M}, T_{N}^{M} C_{N}^{M}, D_{N}^{M}$ are completely positive and normal.

(b2) $S_{N}^{M}$ does not depend on the chosen finite representation: if $(\rho, K)$ is a finite representation of the pair $N \subset M$, one has for $z$ in $Z(M)$

$$
\rho\left(c_{N}(H)\left(c_{M}(H)^{-1} z\right)^{\natural_{N_{H}^{\prime}}}\right)=c_{\rho(N)}(K)\left(c_{\rho(M)}(K)^{-1} \rho(z)\right)^{\natural_{N_{K}^{\prime}}} .
$$

In particular $C_{N}^{M}$ and $D_{N}^{M}$ do not depend on the chosen finite representation.

(b3) The maps $C_{N}^{M}$ and $D_{N}^{M}$ have the same spectral radius.

Proof. The implications (a3) $\Rightarrow(\mathrm{a} 2) \Rightarrow(\mathrm{a} 1)$ are obvious. We show that $(\mathrm{a} 1) \Rightarrow(\mathrm{a} 3)$ : Assume that $M$ is contained in $B(H)$ and that the action of $M$ on $H$ is a finite representation of the pair $N \subset M$, and let $(\pi, K)$ be a finite representation of $M$. By Lemma 2.2, we may assume that $K=e^{\prime}\left(H \otimes K_{n}\right)$ and $\pi(x)=\left(x \otimes 1_{n}\right) e^{\prime}$, where $n$ and $e^{\prime}$ are as in Lemma 2.2. Then $N_{K}^{\prime}=e^{\prime}\left(N_{H}^{\prime} \otimes B\left(K_{n}\right)\right) e^{\prime}$ is a finite 
von Neumann algebra and

$$
c_{\pi(N)}(K)=n e^{\prime}\left(e^{\prime}\right)^{\natural_{N}^{\prime}} c_{N}(H) \otimes 1_{n}
$$

belongs to $Z(\pi(N))$ and is invertible by Lemma 1.2. (The trace on $N_{H}^{\prime} \otimes B\left(K_{n}\right)$ being denoted by $\mathfrak{a}_{n}^{\prime}$.) The assertions (b1) and (b3) are obvious. Let us prove (b2): let $(\rho, K)$ be as in (b2). By Lemma 2.1, it suffices to consider the case where $\rho$ is a spatial isomorphism, a finite dimensional ampliation or the induction by a projection with invertible trace. The first two cases are easy; so assume that $\rho$ is the induction by some projection $e^{\prime}$ in $M_{H}^{\prime}$ such that $\left(e^{\prime}\right)^{t_{H}^{\prime}}$ is invertible, where $\natural_{H}^{\prime}$ denotes the trace on $M_{H}^{\prime}$. Denote by $\natural_{H}^{\prime \prime}$ (resp. $\natural_{K}^{\prime \prime}$ ) the trace on $N_{H}^{\prime}\left(\operatorname{resp} . N_{K}^{\prime}\right)$. Then $K=e^{\prime} H$, and

$$
c_{M_{e^{\prime}}}(K)=e^{\prime}\left(e^{\prime}\right)^{\natural_{H}^{\prime}} \cdot c_{M}(H) \quad \text { and } \quad c_{N_{e^{\prime}}}(K)=e^{\prime}\left(e^{\prime}\right)^{\natural_{H}^{\prime \prime}} \cdot c_{N}(H) .
$$

One gets for every $z$ in $Z(M)$ :

$$
\begin{aligned}
c_{\rho(N)} & (K)\left(c_{\rho(M)}(K)^{-1} \rho(z)\right)^{\natural_{K}^{\prime \prime}} \\
& =e^{\prime} c_{N}(H) e^{\prime \natural_{H}^{\prime \prime}}\left(e^{\prime \natural_{H}^{\prime \prime}}\right)^{-1}\left(e^{\prime}\left(e^{\prime \natural_{H}^{\prime}}\right)^{-1} c_{M}(H)^{-1} z\right)^{\natural_{H}^{\prime \prime}} \\
& =e^{\prime} c_{N}(H)\left(\left(e^{\prime}\left(e^{\prime \natural_{H}^{\prime}}\right)^{-1} c_{M}(H)^{-1} z\right)^{\natural_{H}^{\prime}}\right)^{\natural_{H}^{\prime \prime}} \\
& =e^{\prime} c_{N}(H)\left(c_{M}(H)^{-1} z\right)^{\natural_{H}^{\prime \prime}} .
\end{aligned}
$$

We used Lemma 1.2 in the third line of the computation. The rest of the statement follows now easily.

Definition 2.4. (1) $N$ is said to be of finite index in $M$ if the pair $N \subset M$ satisfies one of the equivalent conditions of Theorem 2.3(a).

(2) If $N$ is of finite index in $M$, then the index of $N$ in $M$, denoted by $[M: N]$, is the common spectral radius of the maps $C_{N}^{M}$ and $D_{N}^{M}$.

(3) (See [3], Definition 3.5.3.) The inclusion of $N$ in $M$ is connected if $Z(N) \cap Z(M)=\mathbb{C}$.

Remark. Suppose that $Z(N)$ and $Z(M)$ are finite dimensional. Then the index above coincides with the index introduced in [3], Definition 3.7.5. Indeed, denote by $p_{1}, \ldots, p_{m}$ (resp. $\left.q_{1}, \ldots, q_{n}\right)$ the minimal central projections of $M$ (resp. $N$ ). By Proposition 3.6.8 of [3], one verifies that the matrices of the maps $S_{N}^{M}$ and $T_{N}^{M}$ written in the bases $p_{1}, \ldots, p_{m}$ and $q_{1}, \ldots, q_{n}$ are precisely the matrices $\widetilde{T}_{N}^{M}$ and $T_{N}^{M}$ of Definition 3.7.5. 
Corollary 2.5. Let $N \subset M \subset L$ be finite, $\sigma$-finite von Neumann algebras.

(1) If $N$ (resp. $M$ ) is of finite index in $M$ (resp. L) then $N$ is of finite index in $L$.

(2) If $N$ is of finite index in $L$ then $N$ (resp. $M$ ) is of finite index in $M$ (resp. L).

(3) Assume that condition (1) or (2) above is satisfied. Then:

(i) $S_{N}^{L}=S_{N}^{M} S_{M}^{L}$;

(ii) $T_{N}^{L}=T_{M}^{L} T_{N}^{M}$;

(iii) $C_{N}^{L}=S_{N}^{M} C_{M}^{L} T_{N}^{M}$;

(iv) $D_{N}^{L}=T_{M}^{L} D_{N}^{M} S_{M}^{L}$.

Proof. (1) follows from a straightforward application of condition (a3) of Theorem 2.3.

(2) Let $H$ be some finite representation of the pair $N \subset L$. We have to prove that the operators $c_{M}(H)^{ \pm 1}$ are bounded. There exists an integer $n \geq 1$ such that $c_{N}(H) \leq n$. By Proposition 5, page 301, of [2], there exist $\xi_{1}, \ldots, \xi_{n}$ in $H$ such that

$$
1=\bigvee_{i=1}^{n} e_{\xi_{i}}^{(N)}
$$

As $N$ is contained in $M$ we get

$$
1=\left(\bigvee_{i=1}^{n} e_{\xi_{l}}^{(M)}\right)^{\natural_{M_{H}^{\prime}}} \leq \sum_{i=1}^{n} c_{M}(H)^{-1}\left(e_{\xi_{l}}^{\left(M^{\prime}\right)}\right)^{\natural_{M}} \leq n c_{M}(H)^{-1},
$$

and hence $c_{M}(H) \leq n$.

Similarly there exists $m \geq 1$ such that $c_{L}(H)^{-1} \leq m$. By applying the proof above to the pair $L_{H}^{\prime} \subset M_{H}^{\prime}$ we get $c_{M}(H)^{-1} \leq m$.

(3)(i) Let $H$ be some finite representation of $L$. One has for every $x$ in $Z(L)$

$$
\begin{aligned}
S_{N}^{M} S_{M}^{L}(x) & =c_{N}(H)\left(c_{M}(H)^{-1} S_{M}^{L}(x)\right)^{\natural_{N}^{\prime}} \\
& =c_{N}(H)\left(c_{M}(H)^{-1} c_{M}(H)\left(c_{L}(H)^{-1} x\right)^{\natural_{M}^{\prime}}\right)^{\natural_{N_{H}^{\prime}}} \\
& =c_{N}(H)\left(c_{L}(H)^{-1} x\right)^{\natural_{N_{H}^{\prime}}} \quad \text { by Lemma } 1.2 \\
& =S_{N}^{L}(x) .
\end{aligned}
$$

(ii) follows immediately from Lemma 1.2, and (iii) and (iv) are straightforward applications of (i) and (ii). 
Let us establish the expressions of the maps $S_{N}^{M}, C_{N}^{M}$ and $D_{N}^{M}$ in the standard representation $L^{2}(M)$ since they will be used frequently: Set $c_{N}=c_{N}\left(L^{2}(M)\right)$ and recall that $c_{M}\left(L^{2}(M)\right)=1$. Then

$$
\begin{aligned}
S_{N}^{M}(z) & =c_{N}\left(L^{2}(M)\right)\left(c_{M}\left(L^{2}(M)\right)^{-1} z\right)^{\natural_{N^{\prime}}} \\
& =c_{N} z^{\natural_{N^{\prime}}} \quad \text { for every } z \text { in } Z(M) .
\end{aligned}
$$

Hence

$$
\begin{aligned}
& C_{N}^{M}(w)=S_{N}^{M} T_{N}^{M}(w)=c_{N}\left(w^{\natural_{M}}\right)^{\natural_{N^{\prime}}} \quad \text { for } w \text { in } Z(N) \text {, and } \\
& D_{N}^{M}(z)=T_{N}^{M} S_{N}^{M}(z)=\left(c_{N} z^{\natural_{N^{\prime}}}\right)^{\natural_{M}} \quad \text { for } z \text { in } Z(M) \text {. }
\end{aligned}
$$

Before considering some examples of such pairs, let us study the case of the pair $M_{H}^{\prime} \subset N_{H}^{\prime}$ where $H$ is some finite representation of $N \subset$ $M$ :

Corollary 2.6. Suppose that $N$ is of finite index in $M$ and let $H$ be some finite representation of the pair $N \subset M$. Then $M_{H}^{\prime}$ is of finite index in $N_{H}^{\prime}$ and

$$
\left[N_{H}^{\prime}: M_{H}^{\prime}\right]=[M: N] \text {. }
$$

Proof. $N_{H}^{\prime}$ is obviously finite and $\sigma$-finite, so $M_{H}^{\prime}$ is of finite index in $N_{H}^{\prime}$. Let $\Gamma: Z(M) \rightarrow Z(M)$ be the multiplication operator by $c_{M}(H)$. Then we get for $z$ in $Z(M)=Z\left(M_{H}^{\prime}\right)$

$$
\begin{aligned}
\Gamma^{-1} D_{N}^{M} \Gamma(z) & =c_{M}(H)^{-1}\left(c_{N}(H)\left(c_{M}(H)^{-1} c_{M}(H) z\right)^{\natural_{N_{H}^{\prime}}}\right)^{\natural_{M}} \\
& =c_{M_{H}^{\prime}}(H)\left(c_{N_{H}^{\prime}}(H)^{-1} z^{\natural_{N_{H}^{\prime}}}\right)^{\natural_{M}} \\
& =C_{M_{H}^{\prime}}^{N_{H}^{\prime}}(z) .
\end{aligned}
$$

Hence the spectral radius of $C_{M_{H}^{\prime}}^{N_{H}^{\prime}}$ is equal to the spectral radius of $D_{N}^{M}$.

ExAmple 2.7. Let $M$ be a finite factor and let $N$ be a von Neumann subalgebra of $M$ containing the identity of $M$. Denote by $\operatorname{tr}_{M}$ the standard trace on $M$, i.e. $x^{\natural_{M}}=\operatorname{tr}_{M}(x) \cdot 1_{M}$ for $x$ in $M$. We will see in $\S 4$ that $Z(N)$ is finite dimensional if $N$ is of finite index in $M$. Let $q_{1}, \ldots, q_{n}$ denote the minimal central projections of $N$. One has

$$
c_{N}=\sum_{i=1}^{n} \operatorname{dim}_{q_{j} N}\left(q_{j} L^{2}(M)\right) q_{j} \quad(\text { see Chapter } 3 \text { of }[3]),
$$

and $D_{N}^{M}$ is the multiplication operator on $\mathbb{C}=Z(M)$ by $\operatorname{tr}_{M}\left(c_{N}\right)$. 
Hence

$$
\begin{aligned}
{[M: N] } & =\operatorname{tr}_{M}\left(c_{N}\right)=\sum_{i=1}^{n} \operatorname{dim}_{q_{j} N}\left(q_{j} L^{2}(M)\right) \operatorname{tr}_{M}\left(q_{j}\right) \\
& =\sum_{i=1}^{n}\left[M_{q_{j}}: N_{q_{j}}\right]
\end{aligned}
$$

since

$$
\left[M_{q_{j}}: N_{q_{j}}\right]=\operatorname{dim}_{q_{j} N}\left(q_{j} L^{2}(M)\right) / \operatorname{dim}_{q_{j} M q_{j}}\left(q_{j} L^{2}(M)\right)
$$

and

$$
\operatorname{dim}_{q_{j} M q_{j}}\left(q_{j} L^{2}(M)\right)=\operatorname{tr}_{M}\left(q_{j}\right)^{-1} .
$$

EXAMPLE 2.8. Let $A$ be an abelian, $\sigma$-finite von Neumann algebra and let $\alpha$ be an automorphism of $A$. Assume that there exists a normal, faithful, semifinite trace $\mu$ on $A$ which is invariant under $\alpha$. Set $M=\operatorname{Mat}_{2}(A)$, and

$$
N=\left\{\left(\begin{array}{cc}
y & 0 \\
0 & \alpha(y)
\end{array}\right) ; y \in A\right\} .
$$

Let $H=L^{2}(A, \mu) \otimes \mathbb{C}^{2}$ and let $u$ be the unitary operator on $L^{2}(A, \mu)$ such that $\alpha=\operatorname{Ad}(u)$. Then

$$
\begin{aligned}
& N_{H}^{\prime}=\left\{\left(\begin{array}{cc}
a & b u^{*} \\
u c & d
\end{array}\right) ; a, b, c, d \in A\right\} \text { is finite, and } \\
&\left(\begin{array}{cc}
a & b u^{*} \\
u c & d
\end{array}\right)^{\natural_{N_{H}^{\prime}}}=\frac{1}{2}\left(\begin{array}{cc}
a+\alpha^{-1}(d) & 0 \\
0 & \alpha(a)+d
\end{array}\right)
\end{aligned}
$$

for $a, b, c, d$ in $A$. Hence $c_{N}(H)=2$ and $c_{M}(H)=1 / 2$, so $N$ is of finite index in $M$. Moreover

$$
\begin{aligned}
& D_{N}^{M}(a)=2 a+\alpha(a)+\alpha^{-1}(a) \text { for } a \text { in } A \cong Z(M), \quad \text { and } \\
& C_{N}^{M}\left(\begin{array}{cc}
a & 0 \\
0 & \alpha(a)
\end{array}\right)=\left(\begin{array}{cc}
D(a) & 0 \\
0 & \alpha(D(a))
\end{array}\right) \text { where } D=D_{N}^{M} .
\end{aligned}
$$

As $D_{N}^{M}(1)=4$, we have $[M: N]=4$.

Finally, remark that the inclusion of $N$ in $M$ is connected if and only if $\alpha$ is ergodic.

The motivation for our definition of index needs the basic construction which is the subject of the next section.

3. The basic construction. Throughout this section $N \subset M$ is a pair of finite, $\sigma$-finite von Neumann algebras, $N$ being of finite index in $M$. 
For $\varphi$ in $M_{*, c}^{+}$, we denote by $\Omega_{\varphi} \in P \subset L^{2}(M)$ the unique positive vector in $L^{2}(M)$ such that

$$
\varphi(x)=\left\langle x \Omega_{\varphi}, \Omega_{\varphi}\right\rangle \text { for } x \text { in } M \text { (see [4]). }
$$

If $\varphi$ is a normal, faithful, finite trace, we denote by $E_{N}^{\varphi}$ the (unique) conditional expectation associated to $\varphi$, and by $e_{N}^{\varphi}$ the orthogonal projection onto $\left[N \Omega_{\varphi}\right]$.

Proposition 3.1. Let $\varphi$ be a normal, faithful, finite trace on $M$. Set $\left\langle M, e_{N}^{\varphi}\right\rangle=\left(M \cup\left\{e_{N}^{\varphi}\right\}\right)^{\prime \prime}$. Then

(1) $\left(e_{N}^{\varphi}\right)^{\natural_{N^{\prime}}}=c_{N}^{-1}$;

(2) $J$ commutes with $e_{N}^{\varphi}$ and $e_{N}^{\varphi} x e_{N}^{\varphi}=E_{N}^{\varphi}(x) e_{N}^{\varphi}$ for $x$ in $M$;

(3) if $\psi$ is another normal, faithful, finite trace on $M$ then the projections $e_{N}^{\varphi}$ and $e_{N}^{\psi}$ are equivalent in $N^{\prime}$ and

$$
\left\langle M, e_{N}^{\varphi}\right\rangle=\left\langle M, e_{N}^{\psi}\right\rangle=J N^{\prime} J .
$$

Moreover, $e_{N}^{\varphi}$ and $e_{N}^{\psi}$ are equivalent in $J N^{\prime} J$.

Proof. (1) By faithfulness of $\varphi$, the vector $\Omega_{\varphi}$ is cyclic and separating for $M$; thus $e_{\Omega_{\varphi}}^{\left(N^{\prime}\right)}=1$. As $e_{N}^{\varphi}=e_{\Omega_{\varphi}}^{(N)}$, we have by definition of $c_{N}=c_{N}\left(L^{2}(M)\right)$

$$
c_{N}\left(e_{N}^{\varphi}\right)^{\natural_{N^{\prime}}}=1 \text {. }
$$

(2) Let $J_{\varphi}$ be the involution on $L^{2}(M)$ extending the map: $x \Omega_{\varphi} \mapsto$ $x^{*} \Omega_{\varphi}$. By Lemma 2.9 of [4], we have $J_{\varphi}=J$. Hence $J e_{N}^{\varphi}=e_{N}^{\varphi} J$ and $e_{N}^{\varphi} x e_{N}^{\varphi}=E_{N}^{\varphi}(x) e_{N}^{\varphi}$ for $x$ in $M$. See [5], $\S 3$.

(3) It follows from (2) that $N=M \cap\left\{e_{N}^{\varphi}\right\}^{\prime}$, and that $\left\langle M, e_{N}^{\varphi}\right\rangle=$ $J N^{\prime} J$. By (1), $e_{N}^{\varphi}$ and $e_{N}^{\psi}$ are equivalent in $N^{\prime}$, as they have the same trace. Finally, they are equivalent in $J N^{\prime} J$ since they commute with $J$.

Thus, in order to define the basic construction for the pair $N \subset M$, we choose an arbitrary normal, faithful, finite trace $\operatorname{tr}$ on $M$, and we set $e_{N}=e_{N}^{\mathrm{tr}}$ and $\left\langle M, e_{N}\right\rangle=J N^{\prime} J$ which is equal to $\left\langle M, e_{N}^{\varphi}\right\rangle$ for any other such trace $\varphi$ on $M$. As $M=J M^{\prime} J$, then $M$ is of finite index in $\left\langle M, e_{N}\right\rangle$ and $\left[\left\langle M, e_{N}\right\rangle: M\right]=[M: N]$. This allows us to iterate the basic construction and we get a tower of finite, $\sigma$-finite von Neumann algebras $\left(M_{k}\right)_{k \geq 0}$ with

$$
M_{0}=N, \quad M_{1}=M, \quad \text { and } \quad M_{k+1}=\left\langle M_{k}, e_{M_{k-1}}\right\rangle=J_{k} M_{k-1}^{\prime} J_{k},
$$

where $J_{k}$ is the involution on the standard representation $L^{2}\left(M_{k}\right)$. 
Recall now that for every normal, faithful, finite trace $\varphi$ on $M$, there exists a unique normal, faithful, semifinite trace $\operatorname{Tr}_{\varphi}^{\prime}$ on $N^{\prime}$ with the following property:

$$
\operatorname{Tr}_{\varphi}^{\prime}\left(e_{N}^{\varphi} y^{\prime} e_{N}^{\varphi}\right)=\left\langle y^{\prime} \Omega_{\varphi}, \Omega_{\varphi}\right\rangle, \quad \text { for } y^{\prime} \text { in } N^{\prime} .
$$

In fact, $\operatorname{Tr}_{\varphi}^{\prime}$ is the trace on $N^{\prime}$ whose spatial derivative (in the sense of [1]) with respect to $\varphi \mid N$ is equal to 1 . Let then $\operatorname{Tr}_{\varphi}$ denote the corresponding trace on $\left\langle M, e_{N}^{\varphi}\right\rangle=\left\langle M, e_{N}\right\rangle=J N^{\prime} J$. It is the unique normal, faithful trace on $\left\langle M, e_{N}\right\rangle$ such that

$$
\operatorname{Tr}_{\varphi}\left(e_{N}^{\varphi} x e_{N}^{\varphi}\right)=\left\langle x \Omega_{\varphi}, \Omega_{\varphi}\right\rangle \text { for } x \text { in }\left\langle M, e_{N}\right\rangle .
$$

Moreover, one has another nice interpretation of the trace $\operatorname{Tr}_{\varphi}:$ By Proposition 3.6.1(v) of [3], the map $\Psi$, from $N$ to the reduced algebra $e_{N}^{\varphi}\left\langle M, e_{N}\right\rangle e_{N}^{\varphi}$ given by $\Psi(y)=y e_{N}^{\varphi}$ for $y$ in $N$ is an isomorphism and it is easily checked that $\operatorname{Tr}_{\varphi}$ is the unique normal trace on $\left\langle M, e_{N}\right\rangle$ such that

$$
\operatorname{Tr}_{\varphi} \mid e_{N}^{\varphi}\left\langle M, e_{N}\right\rangle e_{N}^{\varphi}=\varphi \circ \Psi^{-1}
$$

We are ready to interpret the maps $D_{N}^{M}$ and $S_{M}^{\left\langle M, e_{N}\right\rangle}$ in terms of the traces $\varphi$ and $\operatorname{Tr}_{\varphi}$ on $M$ and $\left\langle M, e_{N}\right\rangle$ respectively. Recall that $D_{*}$ is a map from $M_{*, c}$ to itself and $\widetilde{S}_{*}$ is a map from $M_{*, c}$ to $\left\langle M, e_{N}\right\rangle_{*, c}$, where we set $D=D_{N}^{M}$ and $\widetilde{S}=S_{M}^{\left\langle M, e_{N}\right\rangle}$.

Proposition 3.2. The map $\widetilde{S}_{*}$ is the unique positive, linear, bounded map from $M_{*, c}$ to $\left\langle M, e_{N}\right\rangle_{*, c}$ such that

(1) $\widetilde{S}_{*}(\varphi)=\operatorname{Tr}_{\varphi}$ for every normal, faithful, finite trace $\varphi$ on $M$. Moreover we have for every $\varphi$ in $M_{*, c}$ :

(2) $\widetilde{S}_{*}(\varphi) \mid M=D_{*}(\varphi)$.

Proof. Uniqueness of such a map follows from its continuity, from uniqueness of $\operatorname{Tr}_{\varphi}$ and from density of normal, faithful, finite traces in the positive part of $M_{*, c}$. Let us prove (2) first: we have for any $\varphi$ in $M_{*, c}$ and any $z$ in $Z(M)$

$$
\begin{aligned}
\tilde{S}_{*}(\varphi)(z) & =\varphi\left(S_{M}^{\left\langle M, e_{N}\right\rangle}\left(z^{\natural_{\left\langle M, e_{N}\right\rangle}}\right)\right) \\
& =\varphi\left(\left(J c_{N} J J z^{* \natural_{N^{\prime}}} J\right)^{\natural_{M^{\prime}}}\right) \\
& =\varphi\left(\left(c_{N} z^{\natural_{N^{\prime}}}\right)^{\natural_{M}}\right)=\varphi(D(z))=D_{*}(\varphi)(z),
\end{aligned}
$$

since $c_{\left\langle M, e_{N}\right\rangle}\left(L^{2}(M)\right)=J c_{N}^{-1} J$. 
In order to prove (1), let us fix a normal, faithful, finite trace $\varphi$ on $M$. We establish first the identity:

$$
y^{\natural_{N}}=c_{N}\left(e_{N}^{\varphi} J y^{*} J\right)^{\natural_{N^{\prime}}} \text { for every } y \text { in } N \text {. }
$$

Indeed, set $\theta(y)=c_{N}\left(e_{N}^{\varphi} J y^{*} J\right)^{\natural_{N^{\prime}}}$ for $y$ in $N$. It is easy to check that $\theta$ is linear, positive, bounded and normal, that $\theta\left(y_{1} y_{2}\right)=\theta\left(y_{2} y_{1}\right)$ for $y_{1}, y_{2}$ in $N$, and finally that $\theta(w)=w$ for $w$ in $Z(N)$, since one has for such a $w: e_{N}^{\varphi} J w^{*} J=e_{N}^{\varphi} w$, which implies that $\theta(w)=w$ using Proposition 3.1. By uniqueness of the canonical $Z(N)$-valued trace, we get $\theta=\natural_{N}$, and $(*)$ is proved. Now take $a$ and $b$ in $M$, and set $y=E_{N}^{\varphi}(a) E_{N}^{\varphi}(b)$. Then

$$
\begin{aligned}
\widetilde{S}_{*}(\varphi) & \left(e_{N}^{\varphi}\left(a e_{N}^{\varphi} b\right) e_{N}^{\varphi}\right)=\widetilde{S}_{*}(\varphi)\left(y e_{N}^{\varphi}\right) \\
= & \varphi\left(\left(J c_{N} J\left(y e_{N}^{\varphi}\right)^{\natural_{\left\langle M, e_{N}\right\rangle}}\right)^{\natural_{M}}\right) \\
& =\varphi\left(\left(c_{N}\left(e_{N}^{\varphi} J y^{*} J\right)^{\natural_{N^{\prime}}}\right)^{\natural_{M}}\right) \\
& =\varphi\left(\left(y^{\natural_{N}}\right)^{\natural_{M}}\right)=\varphi(y) \\
& =\left\langle a e_{N}^{\varphi} b \Omega_{\varphi}, \Omega_{\varphi}\right\rangle .
\end{aligned}
$$

Hence $\widetilde{S}_{*}(\varphi)=\operatorname{Tr}_{\varphi}$ by uniqueness of $\operatorname{Tr}_{\varphi}$ and by density of the set of finite sums of elements of the form $a e_{N}^{\varphi} b \quad(a, b$ in $M)$ in the algebra $\left\langle M, e_{N}\right\rangle$ (Lemma 1.1 of [6]).

In the next sections we will need to study the relationships between the maps $C_{N}^{M_{k}}$ and $D_{N}^{M_{k}}$, and the maps $C_{N}^{M}$ and $D_{M_{k-1}}^{M_{k}}$ respectively. The following proposition is the first step in this direction:

Proposition 3.3. Consider a pair $N \subset M$ as above. Then the following identities hold:

(1) $C_{M}^{\left\langle M, e_{N}\right\rangle} T_{N}^{M}=T_{N}^{M} C_{N}^{M}$;

(2) $S_{M}^{\left\langle M, e_{N}\right\rangle} D_{M}^{\left\langle M, e_{N}\right\rangle}=D_{N}^{M} S_{M}^{\left\langle M, e_{N}\right\rangle}$.

Proof. (1) Fix $w$ in $Z(N)$. One gets

$$
\begin{aligned}
C_{M}^{\left\langle M, e_{N}\right\rangle} T_{N}^{M}(w) & =C_{M}^{\left\langle M, e_{N}\right\rangle}\left(w^{\natural_{M}}\right)=S_{M}^{\left\langle M, e_{N}\right\rangle}\left(\left(w^{\natural_{M}}\right)^{\natural_{\left\langle M, e_{N}\right\rangle}}\right) \\
& =S_{M}^{\left\langle M, e_{N}\right\rangle}\left(J\left(w^{* \natural_{M}}\right)^{\natural_{N}} J\right) \\
& =\left(J c_{N}\left(w^{* \natural_{M}}\right)^{\natural_{N^{\prime}}} J\right)^{\natural_{M^{\prime}}} \\
& =\left(c_{N}\left(w^{\natural_{M}}\right)^{\natural_{N}}\right)^{\natural_{M}} \\
& =\left(C_{N}^{M}(w)\right)^{\natural_{M}}=T_{N}^{M} C_{N}^{M}(w) .
\end{aligned}
$$


(2) Similarly one has for $w$ in $Z(N)$

$$
\begin{aligned}
S_{M}^{\left\langle M, e_{N}\right\rangle} D_{M}^{\left\langle M, e_{N}\right\rangle}\left(J w^{*} J\right)=S_{M}^{\left\langle M, e_{N}\right\rangle}\left(\left(\left(J c_{N} w^{*} J\right)^{\natural_{M^{\prime}}}\right)^{\left.\natural_{\left\langle M, e_{N}\right.}\right\rangle}\right) \\
=S_{M}^{\left\langle M, e_{N}\right\rangle}\left(J\left(\left(c_{N} w^{*}\right)^{\natural_{M}}\right)^{\natural_{N^{\prime}}} J\right) \\
=\left(J c_{N}\left(\left(c_{N} w^{*}\right)^{\natural_{M}}\right)^{\natural_{N^{\prime}} ; J}\right)^{\natural_{M^{\prime}}} \\
=\left(c_{N}\left(\left(c_{N} w\right)^{\natural_{M}}\right)^{\natural_{N}{ }^{\prime}}\right)^{\natural_{M}} \\
=D_{N}^{M}\left(\left(c_{N} w\right)^{\natural_{M}}\right) \\
=D_{N}^{M}\left(\left(J c_{N} J J w^{*} J\right)^{\natural_{M^{\prime}}}\right) \\
=D_{N}^{M} S_{M}^{\left\langle M, e_{N}\right\rangle}\left(J w^{*} J\right) .
\end{aligned}
$$

COROLlary 3.4. For every pair $N \subset M$ as above and every positive integer $k$, we have
(1) $C_{N}^{M_{k}}=\left(C_{N}^{M}\right)^{k}$;
(2) $D_{N}^{M_{k}}=\left(D_{M_{k-1}}^{M_{k}}\right)^{k}$.

Proof. (1) By induction on $k$. If $k=1$ it is obvious. Suppose that the assertion is true for some $k$ and for every pair of finite, $\sigma$ finite von Neumann algebras of finite index. Applying the induction hypothesis to the pair $M \subset M_{k+1}$ one gets

$$
\begin{aligned}
C_{N}^{M_{k+1}}= & S_{N}^{M} C_{M}^{M_{k+1}} T_{N}^{M} \quad \text { by Corollary } 2.5 \\
= & S_{N}^{M}\left(C_{M}^{\left\langle M, e_{N}\right\rangle}\right)^{k} T_{N}^{M} \\
= & S_{N}^{M}\left(C_{M}^{\left\langle M, e_{N}\right\rangle}\right)^{k-1} T_{N}^{M} C_{N}^{M} \text { by Proposition } 3.3(1) \\
& \vdots \\
= & S_{N}^{M} C_{M}^{\left\langle M, e_{N}\right\rangle} T_{N}^{M}\left(C_{N}^{M}\right)^{k-1} \\
= & S_{N}^{M} T_{N}^{M}\left(C_{N}^{M}\right)^{k}=\left(C_{N}^{M}\right)^{k+1} .
\end{aligned}
$$

The assertion (2) is proved in the same way.

Corollary 3.5. Let $N \subset M$ and $k$ as above; one has

(1) $C^{k}(1)=c_{N}\left(L^{2}\left(M_{k}\right)\right)$, where $C=C_{N}^{M}$;

(2) $\left[M_{k}: N\right]=[M: N]^{k}$;

(3) $[M: N] \geq 1$.

Proof. (1) $C^{k}(1)=C_{N}^{M_{k}}(1)=c_{N}\left(L^{2}\left(M_{k}\right)\right)$ when computed in the finite representation $L^{2}\left(M_{k}\right)$. 
(2)

$$
\begin{aligned}
{\left[M_{k}: N\right] } & =\lim _{m}\left\|\left(C_{N}^{M^{k}}\right)^{m}\right\|^{1 / m}=\lim _{m}\left\|C^{m k}\right\|^{1 / m} \\
& =\lim _{m}\left(\left\|C^{m k}\right\|^{1 / m k}\right)^{k}=[M: N]^{k} .
\end{aligned}
$$

(3) By Proposition 3.1 we have $c_{N}\left(L^{2}\left(M_{k}\right)\right)^{-1} \leq 1$, hence $C^{k}(1) \geq$ 1 , and $\left\|C^{k}\right\|^{1 / k} \geq 1$ for every $k$ since $C$ is completely positive.

4. The case of pairs with atomic centers. We assume here that $N \subset M$ is a pair of finite, $\sigma$-finite von Neumann algebras with atomic centers (see [10], Definition III.5.9). It is equivalent to say that $N$ and $M$ are finite or countable direct sums of finite factors. The purpose of the present section is to prove that the index defined above coincides with the index introduced in $\S 2.1$ of [3]. We denote by $\operatorname{Min}(M)$ the set of minimal central projections of $M$ and by $\operatorname{Min}(N)$ the corresponding set of $N$. If $p$ and $p^{\prime}$ belong to $\operatorname{Min}(M)$, we say that they are neighbors if there exists $q$ in $\operatorname{Min}(N)$ such that $p q \neq 0$ and $p^{\prime} q \neq 0$. We denote by $W_{p}$ the set of neighbors of $p \in \operatorname{Min}(M)$.

LEMMA 4.1. There exists a positive integer $n$ such that for every $p$ in $\operatorname{Min}(M)$ one has

$$
\operatorname{card}\left(W_{p}\right) \leq n
$$

Moreover, the projection $z(p)=\sum_{e \in W_{p}}$ e is the support of $D_{N}^{M}(p)$ in $Z(M)$.

Proof. Set $Q_{p}=\{q \in \operatorname{Min}(N) ; p q \neq 0\}$ for $p$ in $\operatorname{Min}(M)$. Then $\sum_{q \in Q_{p}} q$ is the central support of $p$ in $N^{\prime}$, and using Lemma 3.6.7 of [3], we see that:

$$
c_{N} p^{\natural_{N}}=\sum_{q \in Q_{p}}\left(\lambda_{p, q}^{2} / c_{p, q}\right) q,
$$

where $\lambda_{p, q}^{2}=\left[M_{p q}: N_{p q}\right] \geq 1$ and $c_{p, q}=\operatorname{tr}_{p M}(p q)$. We get for $q$ in $Q_{p}$

$$
\left\|c_{N}\right\| \cdot q \geq c_{N} p^{\natural_{N^{\prime}}} q \geq\left(1 / c_{p, q}\right) q ; \text { hence } c_{p, q}\left\|c_{N}\right\| \geq 1 \text {. }
$$

But

$$
1=\sum_{q \in \operatorname{Min}(N)} \operatorname{tr}_{p M}(p q)=\sum_{q \in Q_{p}} c_{p, q},
$$

which implies $\operatorname{card}\left(Q_{p}\right) \leq\left\|c_{N}\right\|$. Similarly, we have

$$
\operatorname{card}\left\{p^{\prime} \in \operatorname{Min}(M) ; p^{\prime} q \neq 0\right\} \leq\left\|c_{N}^{\natural_{M}}\right\|
$$


for every $q \in Q_{p}$, since $c_{N}^{\natural_{M}}=c_{M^{\prime}}\left(L^{2}\left(N^{\prime}\right)\right)$. Hence $\operatorname{card}\left(W_{p}\right) \leq$ $\left\|c_{N}\right\| \cdot\left\|c_{N}^{\natural_{M}}\right\|$.

Finally, as $D_{N}^{M}(p)=\left(c_{N} p^{\natural_{N^{\prime}}}\right)^{\natural_{M}}$, the last claim is easy to check.

LEMMA 4.2. There exists a normal, faithful, finite trace $\varphi$ on $M$ with the following property: for every $x$ in $\left\langle M, e_{N}\right\rangle$, there exists a unique $y$ in $M$ such that

$$
x e_{N}^{\varphi}=y e_{N}^{\varphi} .
$$

In particular, $\left\langle M, e_{N}\right\rangle=\left\{\sum_{j=1}^{n} a_{j} e_{N}^{\varphi} b_{j} ; a_{j}, b_{j} \in M\right\}$. (See Lemma 3.6.3 of [3].)

Proof. The proof is decomposed into two parts:

(1) We show that there exists a normal, faithful, finite trace $\varphi$ on $M$ and a positive number $c$ such that

$$
D_{*}(\varphi) \leq c \varphi .
$$

Indeed, if $Z(N)$ or $Z(M)$ is finite dimensional then they are both finite dimensional by Lemma 4.1, and any faithful trace on $M$ satisfies $(*)$. We assume that $Z(N)$ and $Z(M)$ are infinite dimensional and that the inclusion of $N$ in $M$ is connected. Then fix an arbitrary projection $p_{0}$ in $\operatorname{Min}(M)$ and set $V_{0}=\left\{p_{0}\right\}$ and $V_{1}=W_{p_{0}} \backslash V_{0}$. For $k \geq 1$, we set

$$
V_{k+1}=\left\{p \in \operatorname{Min}(M) ; p \notin \bigcup_{j=0}^{k} V_{j} \text { and } \exists p^{\prime} \in V_{k} \text { such that } p^{\prime} \in W_{p}\right\} \text {. }
$$

By connectedness of the pair $N \subset M$, we get that $\operatorname{Min}(M)$ is the union of the $V_{k}$ 's and that $V_{k} \neq \varnothing$ for every $k$ (since if $V_{k+1}=\varnothing$ for some $k \geq 0$, then the projection $z=\sum_{j=0}^{k} \sum_{e \in V_{j}} e$ belongs to $Z(N) \cap Z(M)$, hence equals 1$)$.

Moreover, if $n$ denotes the integer given by Lemma 4.1, it is easy to verify that:

(i) $\operatorname{card}\left(V_{k}\right) \leq n^{k}$ for every $k$;

(ii) for every $k$ and for every $p$ in $V_{k}$ one has: $W_{p} \subset \bigcup_{j=k-1}^{k+1} V_{j}$. Then we set $\varphi\left(p_{0}\right)=1 / 2$ and for $p \in V_{k}: \varphi(p)=n^{-k} k^{-2}$. Thus there exists $c^{\prime}>0$ such that

$$
\sum_{p^{\prime} \in W_{p}} \varphi\left(p^{\prime}\right) \leq c^{\prime} \varphi(p) \text { and } \sum_{p} \varphi(p) \leq \frac{1}{2}+\sum_{k \geq 1} k^{-2}<\infty,
$$

for every $p$ in $\operatorname{Min}(M)$. 
As $D(p) \leq\left\|c_{N}\right\| z(p)$, the trace $\varphi$ above satisfies $(*)$ with $c=$ $\left\|c_{N}\right\| c^{\prime}$.

(2) We take $\varphi$ as in (1) and we set $\tilde{\varphi}=\widetilde{S}_{*}(\varphi)=\operatorname{Tr}_{\varphi} \in\left\langle M, e_{N}\right\rangle_{*, c}$ (see Proposition 3.2). Let $E_{M}$ denote the conditional expectation from $\left\langle M, e_{N}\right\rangle$ onto $M$ associated to $\tilde{\varphi}$. We show that $E_{M}\left(e_{N}^{\varphi}\right)$ is invertible. Indeed, let $\Omega_{\varphi}$ and $\Omega_{\tilde{\varphi}} \in P \subset L^{2}(M)$ be the vectors associated to $\varphi$ and to $\tilde{\varphi} \mid M=D_{*}(\varphi)$ respectively. We get for every $a$ in $M$

$$
\begin{aligned}
& \left\langle E_{M}\left(e_{N}^{\varphi}\right) a \Omega_{\tilde{\varphi}}, a \Omega_{\tilde{\varphi}}\right\rangle=\tilde{\varphi}\left(E_{M}\left(e_{N}^{\varphi} a a^{*}\right)\right)=\tilde{\varphi}\left(e_{N}^{\varphi} a a^{*}\right) \\
& \quad=\left\langle a a^{*} \Omega_{\varphi}, \Omega_{\varphi}\right\rangle \quad \text { by Proposition } 3.2 \\
& \quad=\varphi\left(a^{*} a\right) \\
& \quad \geq c^{-1} D_{*}(\varphi)\left(a^{*} a\right)=c^{-1}\left\langle a \Omega_{\tilde{\varphi}}, a \Omega_{\tilde{\varphi}}\right\rangle .
\end{aligned}
$$

We end the proof as in Lemma 3.6.3 of [3].

Before stating and proving the main result of this section, let us recall the definition of the ring-theoretic basic construction proposed in $\S 2.1$ of [3]: We associate to the pair $N \subset M$ the pair $M \subset \operatorname{End}_{N}^{r}(M)$, where $\operatorname{End}_{N}^{r}(M)$ is the algebra of endomorphisms of $M$ viewed as a right $N$-module; $M$ is identified with a subalgebra of $\operatorname{End}_{N}^{r}(M)$, each $x \in M$ being identified with the left multiplication operator $(y \mapsto x y) \in \operatorname{End}_{N}^{r}(M)$. If $L$ is a right $N$-module we denote by $\operatorname{rk}(L \mid N)$ the smallest possible number of generators of $L$ as a right $N$-module.

THEOREM 4.3. Let $N \subset M$ be a pair of finite, $\sigma$-finite von Neumann algebras with atomic centers, $N$ being of finite index in $M$. Then

(1) $\left\|C_{N}^{M}\right\| \leq \operatorname{rk}(M \mid N) \leq\left\|C_{N}^{M}\right\|+1$;

(2) $M \otimes_{N} M \cong\left\langle M, e_{N}\right\rangle$ as $N$-bimodules and $\operatorname{End}_{N}^{r}(M) \cong\left\langle\left\langle M, e_{N}\right\rangle\right.$ as $\mathbb{C}$-algebras;

(3)

$$
\begin{aligned}
{[M: N] } & =\limsup _{k \rightarrow \infty}\left[\operatorname{rk}\left(M^{\otimes_{N}^{k}} \mid N\right)\right]^{1 / k} \\
& =\limsup _{k \rightarrow \infty}\left[\operatorname{rk}\left(M_{k} \mid N\right)\right]^{1 / k},
\end{aligned}
$$

where $M^{\otimes_{N}^{k}}=M \otimes_{N} M \cdots \otimes_{N} M, k$ times.

Proof. (1) We have by definition $C_{N}^{M}(1)=c_{N}\left(L^{2}(M)\right)=c_{N}$. Set $r=\operatorname{rk}(M \mid N)$. Then there exist $\xi_{1}, \ldots, \xi_{r}$ in $L^{2}(M)$ such that the 
subspace generated by the $N \xi_{j}$ 's is dense in $L^{2}(M)$. This means that

$$
1=\bigvee_{j=1}^{r} e_{\xi}^{(N)}
$$

Thus:

$$
1=\left(\bigvee_{j=1}^{r} e_{\xi_{j}}^{(N)}\right)^{\natural_{N^{\prime}}} \leq \sum_{j=1}^{r} c_{N}^{-1}\left(e_{\xi_{j}}^{\left(N^{\prime}\right)}\right)^{\natural_{N}} \leq r c_{N}^{-1},
$$

which implies that

$$
\left\|C_{N}^{M}(1)\right\| \leq r=\operatorname{rk}(M \mid N) .
$$

As $C_{N}^{M}$ is completely positive, we have $\left\|C_{N}^{M}\right\|=\left\|C_{N}^{M}(1)\right\|$, and the first inequality is proved.

In order to prove the second one, let $\varphi$ be a normal, faithful, finite trace on $M$ as in Lemma 4.2, and let $m$ be the integer such that

$$
\left\|c_{N}\right\| \leq m \leq\left\|c_{N}\right\|+1
$$

We are going to exhibit a basis $\left\{v_{i} ; i=1, \ldots, m\right\}$ of $M$ over $N$ with the following properties:

(a) $E_{N}^{\varphi}\left(v_{i}^{*} v_{j}\right)=0$ if $i \neq j$;

(b) $f_{i}=E_{N}^{\varphi}\left(v_{i}^{*} v_{i}\right)$ is a projection in $N, v_{i} f_{i}=v_{i}$, and $E_{N}^{\varphi}\left(v_{i}^{*} x\right)=$ $f_{i} E_{N}^{\varphi}\left(v_{i}^{*} x\right)$ for every $i$ and every $x$ in $M$;

(c) every $x$ in $M$ has a unique expansion

$$
x=\sum_{i=1}^{m} v_{i} y_{i} \quad \text { with } y_{i} \text { in } N
$$

in fact $v_{i} y_{i}=v_{i} E_{N}^{\varphi}\left(v_{i}^{*} x\right)$.

Such a basis $\left\{v_{i}\right\}$ is called a Pimsner-Popa basis of $M$ over $N$; see [6] and Theorem 3.6.4 of [3].

Remark first that for every $q$ in $\operatorname{Min}(N)$ we have

$$
\operatorname{tr}_{J q J\left(M, e_{N}\right\rangle}\left(e_{N}^{\varphi} J q_{j}\right) \geq m^{-1},
$$

since $\left(e_{N}^{\varphi}\right)^{\natural_{N^{\prime}}}=c_{N}^{-1}$. Hence for every $q$ in $\operatorname{Min}(N)$ there exist $m$ pairwise orthogonal projections $r_{i}(q)$ such that

$$
r_{i}(q) \precsim e_{N}^{\varphi} J q J \quad \text { for every } i, \text { and } \sum_{i=1}^{m} r_{i}(q)=J q J
$$


If $r_{i}=\sum_{q \in \operatorname{Min}(N)} r_{i}(q)$, then the projections $r_{i}$ 's are pairwise orthogonal, their sum equals 1 and $r_{i} \precsim e_{N}^{\varphi}$ for every $i$. Then there exist $m$ partial isometries $w_{i} \in\left\langle M, e_{N}\right\rangle$ with

$$
w_{i}^{*} w_{i} \leq e_{N}^{\varphi} \quad \text { and } \quad \sum_{i=1}^{m} w_{i} w_{i}^{*}=1
$$

By Lemma 4.2 there exist $v_{1}, \ldots, v_{m}$ in $M$ such that $w_{i}=v_{i} e_{N}^{\varphi}$ for every $i$. It is easily verified that the collection $\left\{v_{i}\right\}$ is a PimsnerPopa basis of $M$ over $N$ : see the proof of Theorem 3.6.4 of [3]. This implies that $\operatorname{rk}(M \mid N) \leq m$, and (1) is proved.

Finally (2) follows from the existence of the Pimsner-Popa basis above, and assertion (3) follows from assertions (1) and (2) and from Corollary 3.4(1).

5. Markov traces. We return to the general case where $N \subset M$ is a pair of finite, $\sigma$-finite von Neumann algebras with the same identity, $N$ being of finite index in $M$. We set as usual $C=C_{N}^{N}, D=D_{N}^{M}$ and $\tilde{S}=S_{M}^{\left\langle M, e_{N}\right\rangle}$. (Recall that $F_{*}(\varphi)=\varphi \circ F \circ$ ६.) Let us recall Definition 3.7.1 of [3]:

Definition 5.1. A normal, faithful, finite trace $\varphi$ on $M$ is a Markov trace of modulus $\beta$ for the pair $N \subset M$ if it extends to a normal, faithful, finite trace $\tilde{\varphi}$ on $\left\langle M, e_{N}\right\rangle$ such that

$$
\beta \tilde{\varphi}\left(e_{N}^{\varphi} x\right)=\varphi(x) \text { for } x \text { in } M .
$$

Proposition 5.2. (1) Let $\varphi$ be a normal, faithful, finite trace on $M$. Then $\varphi$ is a Markov trace of modulus $\beta$ for the pair $N \subset M$ if and only if

$$
D_{*}(\varphi)=\beta \varphi .
$$

(2) Suppose that $\varphi$ is a Markov trace of modulus $\beta$ for the pair $N \subset M$. Then its extension to $\left\langle M, e_{N}\right\rangle$ is equal to $\beta^{-1} \tilde{S}_{*}(\varphi)$ and it is a Markov trace of modulus $\beta$ for the pair $M \subset\left\langle M, e_{N}\right\rangle$.

Proof. (1) Assume that $\varphi$ is a Markov trace of modulus $\beta$. We prove that its extension $\tilde{\varphi}$ is equal to $\beta^{-1} \widetilde{S}_{*}(\varphi)$. Indeed, if $a, b \in M$, we have

$$
\begin{aligned}
\beta \tilde{\varphi}\left(e_{N}^{\varphi}\left(a e_{N}^{\varphi} b\right) e_{N}^{\varphi}\right) & =\beta \tilde{\varphi}\left(e_{N}^{\varphi} E_{N}^{\varphi}(a) b\right) \\
& =\varphi\left(E_{N}^{\varphi}(a) b\right)=\left\langle a e_{N}^{\varphi} b \Omega_{\varphi}, \Omega_{\varphi}\right\rangle .
\end{aligned}
$$

Hence the normal, faithful trace $\beta \tilde{\varphi}$ satisfies

$$
\beta \tilde{\varphi}\left(e_{N}^{\varphi} x e_{N}^{\varphi}\right)=\left\langle x \Omega_{\varphi}, \Omega_{\varphi}\right\rangle \text { for } x \text { in }\left\langle M, e_{N}\right\rangle .
$$


By Proposition 3.2 we get $\beta \tilde{\varphi}=\operatorname{Tr}_{\varphi}=\tilde{S}(\varphi)$, and

$$
D_{*}(\varphi)=\widetilde{S}_{*}(\varphi)|M=\beta \tilde{\varphi}| M=\beta \varphi .
$$

Conversely, if $\varphi$ is an eigenvector of $D_{*}$ with eigenvalue $\beta$, set $\tilde{\varphi}=$ $\beta^{-1} \widetilde{S}_{*}(\varphi)$. Then

$$
\beta \tilde{\varphi}\left(e_{N}^{\varphi} x\right)=\widetilde{S}_{*}(\varphi)\left(e_{N}^{\varphi} x\right)=\left\langle x \Omega_{\varphi}, \Omega_{\varphi}\right\rangle=\varphi(x) \text { for } x \text { in } M .
$$

Moreover $\tilde{\varphi}$ extends $\varphi$ :

$$
\tilde{\varphi}\left|M=\beta^{-1} \widetilde{S}_{*}(\varphi)\right| M=\beta^{-1} D_{*}(\varphi)=\varphi \quad \text { by assumption. }
$$

(2) By (1), we need only check that $\tilde{\varphi} \circ D_{M}^{\left\langle M, e_{N}\right\rangle}=\beta \tilde{\varphi}$. Using Proposition 3.3(1), we get

$$
\begin{aligned}
\tilde{\varphi} \circ D_{M}^{\left\langle M, e_{N}\right\rangle} & =\beta^{-1} \varphi \circ S_{M}^{\left\langle M, e_{N}\right\rangle} D_{M}^{\left\langle M, e_{N}\right\rangle} \\
& =\beta^{-1} \varphi \circ D_{N}^{M} S_{M}^{\left\langle M, e_{N}\right\rangle}=\varphi \circ \widetilde{S}=\beta \tilde{\varphi} .
\end{aligned}
$$

COROLlary 5.3. Suppose that there exists a normalized Markov trace $\operatorname{tr}_{M}$ of modulus [M:N] for the pair $N \subset M$. If $\left(M_{k}\right)$ denotes the tower given by iterating the basic construction, let $\operatorname{tr}_{M_{k}}$ be the Markov trace of modulus $[M: N]$ extending $\operatorname{tr}_{M}$.

Then for every $k$, the trace $\operatorname{tr}_{M_{k}}$ is a normalized Markov trace of modulus $\left[M_{k}: N\right]=[M: N]^{k}$ for the pair $N \subset M_{k}$.

Proof. Set $D_{k}=D_{M_{k-1}}^{M_{k}}$; by Corollary 3.4 we have $\left(D_{k}\right)^{k}=D_{N}^{M_{k}}$. As $\operatorname{tr}_{M_{k}} \circ D_{k}=[M: N] \operatorname{tr}_{M_{k}}$, we see that

$$
\operatorname{tr}_{M_{k}} \circ D_{N}^{M_{k}}=[M: N]^{k} \operatorname{tr}_{M_{k}}=\left[M_{k}: N\right] \operatorname{tr}_{M_{k}} \text { by Corollary 3.5. }
$$

REMARK. Let $p$ be a non-zero projection of $Z(N) \cap Z(M)$. Then the reduced algebra $N p$ is of finite index in $M p$ and $[M p: N p] \leq$ $[M: N]$ since $C_{N p}^{M p}$ is the restriction of $C_{N}^{M}$ to $Z(N p)=Z(N) p$. If $\varphi$ is a Markov trace of modulus $[M: N]$ for $N \subset M$, then the corresponding trace $\varphi_{p}$ on $M p$ is a Markov trace of modulus $[M: N]$ for $N p \subset M p$. Hence $[M p: N p]=[M: N]$ for any non-zero projection $p$ of $Z(N) \cap Z(M)$.

Of course, such a condition is almost never fulfilled in the nonconnected case. This shows that connectedness of the pair $N \subset M$ is a reasonable hypothesis for the study of Markov traces of modulus $[M: N]$. 
Definition 5.4. Let $A$ be an abelian von Neumann algebra and let $F$ be some positive, normal endomorphism of $A$. We say that $F$ is irreducible if no $\sigma$-weakly closed ideal of $A$, distinct from $\{0\}$ and $A$, is invariant under $F$.

Remark. Let $A$ and $F$ be as above. The predual $A_{*}$ of $A$ is a Banach lattice, and a subspace $V$ of $A_{*}$ is solid (in the sense that if $\varphi \in V$ and $\psi \in A_{*}$ and if $|\psi| \leq|\varphi|$ then $\left.\psi \in V\right)$ if and only if it is invariant under the action of $A$, i.e. $a \cdot \varphi \in V$ for $a$ in $A$ and $\varphi$ in $V$. Hence, using Theorem III.2.7 of [10], it easy to verify that $F$ is irreducible if and only if its preadjoint $F_{*}$ is irreducible in the sense of [9], page 269, i.e. no closed solid subspace, distinct from $\{0\}$ and $A_{*}$, is invariant under $F_{*}$.

The following result provides a link between connectedness of the pair $N \subset M$ and Perron-Frobenius theory for irreducible positive maps on Banach lattices:

Proposition 5.5. For a pair $N \subset M$ as above, the following conditions are equivalent:

(1) the inclusion of $N$ in $M$ is connected;

(2) the map $C_{N}^{M}$ is irreducible;

(3) the map $D_{N}^{M}$ is irreducible.

Proof. Connectedness of the pair $N \subset M$ is equivalent to connectedness of the pair $M^{\prime} \subset N^{\prime} \subset B\left(L^{2}(M)\right)$. As $C_{M^{\prime}}^{N^{\prime}}=D_{N}^{M}$ (see the proof of Corollary 2.6), we need only prove equivalence between (1) and (3). Thus assume first that $Z(N) \cap Z(M) \neq \mathbb{C}$, let $p$ be some non-trivial projection of $Z(N) \cap Z(M)$ and set $I=Z(M) p$. As $D(z p)=D(z) p$ for $z$ in $Z(M)$, we see that $D$ is not irreducible. Suppose now that the pair is connected, and let $I \neq\{0\}$ be some $\sigma$-weakly closed invariant ideal of $Z(M)$. Denote by $p$ the support of $I: I=Z(M) p$. Hence $D(p)=D(p) p$, and by faithfulness of $\natural_{M}$, we get $p^{\natural_{N}}(1-p)=0$. Let $q$ be the support of $p^{\natural_{N^{\prime}}}$ in $Z(N)$. We are going to prove that $q=p$. We need only show that $q(1-p)=0$, because $q$ is also the central support of $p$ in $N^{\prime}$. For every integer $n \geq 1$ let $q_{n} \in Z(N)$ be the spectral projection of $p^{\natural_{N^{\prime}}}$ corresponding to the interval $[1 / n, \infty)$. The sequence $\left(q_{n}\right)$ increases to $q$ and as

$$
p^{\natural_{N^{\prime}}} q_{n} \geq(1 / n) q_{n},
$$

there exists a sequence $\left(x_{n}\right)$ in $Z(N)_{+}$such that $x_{n}=x_{n} q_{n}$ and 
$x_{n} p^{\natural_{N^{\prime}}}=q_{n}$ for every $n$. Thus $q_{n}(1-p)=x_{n} p^{\natural_{N^{\prime}}}(1-p)=0$ so

$$
q(1-p)=\bigvee_{n} q_{n}(1-p)=0 \text {. }
$$

By classical Perron-Frobenius theory, if the centers $Z(N)$ and $Z(M)$ are finite dimensional then the pair $N \subset M$ admits a unique Markov trace of modulus $[M: N]$, provided that the inclusion is connected. See [3], Corollary 3.7.4. In the infinite dimensional case, we will see that Markov traces may or may not exist. We need the following result:

Proposition 5.6. Suppose that the inclusion of $N$ in $M$ is connected and that $C_{N}^{M}$ or $D_{N}^{M}$ admits a positive eigenvector associated to the eigenvalue $[M: N]$. Then any non-negative, normalized eigenvector of $D_{*}$, if it exists, is a normalized Markov trace of modulus $[M: N]$, and such a trace is unique.

Proof. In any case, we can assume that $D$ admits a positive eigenvector associated to $[M: N]$, since if $0 \neq w_{0} \in Z(N)_{+}$is such that $C\left(w_{0}\right)=[M: N] w_{0}$ then $z_{0}=w_{0}^{\natural_{M}} \neq 0$ and $D\left(z_{0}\right)=[M: N] z_{0}$. Let

$$
V=\left\{\varphi \in M_{*, c} ;|\varphi|\left(z_{0}\right)=0\right\} \text {. }
$$

It is easy to see that $V$ is a closed solid subspace of $M_{*, c}$. Moreover, if $\varphi \in V, \varphi=\varphi_{1}+i \varphi_{2}$ with $\varphi_{j}=\varphi_{j}^{*}$ then $\varphi_{j} \in V$ and

$$
\left|D_{*}\left(\varphi_{j}\right)\right|\left(z_{0}\right) \leq D_{*}\left(\left|\varphi_{j}\right|\right)\left(z_{0}\right)=[M: N]\left|\varphi_{j}\right|\left(z_{0}\right)=0,
$$

which shows that $D_{*}(\varphi)=D_{*}\left(\varphi_{1}\right)+i D_{*}\left(\varphi_{S}\right)$ belongs to $V$. By the irreducibility of $D$, we have $V=\{0\}$ since $z_{0} \neq 0$. Now, if $\varphi$ is an element of $M_{*, c}$ such that $\varphi \geq 0, \varphi(1)=1$ and $D_{*}(\varphi)=\beta \varphi$ then

$$
\beta \varphi\left(z_{0}\right)=D_{*}(\varphi)\left(z_{0}\right)=[M: N] \varphi\left(z_{0}\right),
$$

which proves that $\beta=[M: N]$ since $\varphi\left(z_{0}\right)>0$. Moreover, $\varphi$ is faithful: indeed, let $I_{\varphi}=\left\{z \in Z(M): \varphi\left(z^{*} z\right)=0\right\}$. Then $I_{\varphi}$ is a $\sigma$-weakly closed ideal of $Z(M)$ and it is invariant under $D$ : if $Z$ is in $I_{\varphi}$ we get

$$
\varphi\left(D(z)^{*} D(z)\right) \leq\|D\| \varphi\left(D\left(z^{*} z\right)\right)=\|D\|[M: N] \varphi\left(z^{*} z\right)=0 .
$$

Since $I_{\varphi} \neq Z(M)$, we have $I_{\varphi}=\{0\}$.

Finally, if $\varphi_{1}, \varphi_{2}$ are Markov traces of modulus [M:N], set $\psi=\varphi_{1}-\varphi_{2}$. Let $\psi=\psi_{+}-\psi_{-}$be the Jordan decomposition of $\psi$. Then

$$
[M: N]|\psi|=\left|D_{*}(\psi)\right| \leq D_{*}(|\psi|)
$$


but $D_{*}(|\psi|)\left(z_{0}\right)-[M: N]|\psi|\left(z_{0}\right)=0$, so $D_{*}(|\psi|)=[M: N]|\psi|$ and $\psi_{+}$and $\psi_{-}$are both eigenvectors of $D_{*}$ with eigenvalue $[M: N]$. As their supports are orthogonal and as $\psi(1)=0$, we see that $\psi=0$.

EXAMPLE 5.7. Let us consider the pair $N \subset M$ of Example 2.7: $M=\operatorname{Mat}_{2}(A)$ with $A$ abelian and

$$
N=\left\{\left(\begin{array}{cc}
a & 0 \\
0 & \alpha(a)
\end{array}\right) ; a \in A\right\},
$$

where $\alpha$ is an ergodic automorphism of $A$. Recall that $[M: N]=4$, and that $D(a)=2 a+\alpha(a)+\alpha^{-1}(a)$ for every $a$ in $A \cong Z(M)$. Since 1 is a positive eigenvector of $D$ associated to $4, D_{*}$ has at most one eigenvector associated to 4 which is normalized. As $\alpha \circ D=D \circ \alpha$, if $\varphi$ is such a vector, then $\varphi|A \circ \alpha=\varphi| A$. Consequently, if $A=l^{\infty}(\mathbb{Z})$ and if $\alpha$ is the shift automorphism then there is no Markov trace for the pair $N \subset M$. If $S^{1}$ denotes the unit circle, if $A=L^{\infty}\left(S^{1}\right)$ and if $\alpha$ is some irrational rotation, then the trace on $M$ defined by Lebesgue measure on $S^{1}$ is the unique Markov trace for the pair $N \subset M$. These examples were suggested to me by G. Skandalis.

We generalize now Theorem 2.6 of [7]:

LEMMA 5.8. Suppose that there exists a normalized Markov trace $\operatorname{tr}_{M}$ of modulus [M:N] for the pair $N \subset M$. Let $L$ be a von Neumann algebra containing $M$ and let $\operatorname{tr}_{L}$ be a normalized, normal, faithful, finite trace on $L$ extending $\operatorname{tr}_{M}$. Finally let $e$ be some projection of $L$. Then the following conditions are equivalent:

(1) There exists a spatial isomorphism $\pi$ from $\left\langle M, e_{N}\right\rangle$ onto $L$ such that:

$$
\pi(x)=x \quad \text { for } x \text { in } M \text { and } \pi\left(e_{N}\right)=e,
$$

where $e_{N}=e_{N}^{\operatorname{tr}^{M}}$

(2) L and $e$ have the following properties:

(i) the central support of $e$ in $L$ is equal to 1 ;

(ii) exe $=E_{N}(x) e$ for $x$ in $M$;

(iii) $E_{M}(e)=\beta^{-1}$, where $\beta=[M: N]$;

(iv) $L=(M \cup\{e\})^{\prime \prime}$.

Proof. The implication (1) $\Rightarrow(2)$ is clear. Assume that (2) holds. Denote by $M e_{N} M$ (resp. $M e M$ ) the subset of $\left\langle M, e_{N}\right\rangle$ (resp. L) constituted by all finite sums of elements of the form $a e_{N} b$ (resp. $a e b$ ) with $a, b$ in $M$. The $M e_{N} M$ (resp. $M e M$ ) is a strongly dense ${ }^{*}$-subalgebra of $\left\langle M, e_{N}\right\rangle$ (resp. $L$ ), by properties (i), (ii) and 
(iv): see [3], Proposition 3.6.1. Denote by $\Omega \in L^{2}\left(\left\langle M, e_{N}\right\rangle\right)$ and by $\Omega^{\prime} \in L^{2}(L)$ the positive vectors associated to $\operatorname{tr}_{\left\langle M, e_{N}\right\rangle}$ and to $\operatorname{tr}_{L}$ respectively. Then the subspaces $M e_{N} M \Omega$ and $M e M \Omega^{\prime}$ are dense in $L^{2}\left(\left\langle M, e_{N}\right\rangle\right)$ and in $L^{2}(L)$ respectively. Using property (iii) one verifies that the map

$$
\sum_{j=1}^{n} a_{j} e_{N} b_{j} \Omega \mapsto \sum_{j=1}^{n} a_{j} e b_{j} \Omega^{\prime}
$$

is an isometry from $M e_{N} M \Omega$ to $M e M \Omega^{\prime}$. Thus it extends to a surjective isometry $u$ from $L^{2}\left(\left\langle M, e_{N}\right\rangle\right)$ onto $L^{2}(L)$. Then we set

$$
\pi(x)=u x u^{*} \text { for } x \text { in }\left\langle M, e_{N}\right\rangle \text {. }
$$

We get

$$
\pi\left(\sum_{j=1}^{n} a_{j} e_{N} b_{j}\right)=\sum_{j=1}^{n} a_{j} e b_{j}
$$

for $a_{1}, \ldots, a_{n}, b_{1}, \ldots, b_{n}$ in $M$; it is easy to check that $\pi(x)=x$ for $x$ in $M$.

Assume henceforth that there exists a normalized Markov trace $\operatorname{tr}_{M}$ of modulus $\beta=[M: N]$ for the pair $N \subset M$. Denote by $E_{M_{k}}$ the conditional expectation from $M_{k+1}$ onto $M_{k}$ associated to the Markov trace $\operatorname{tr}_{M_{k+1}}$ and let $\left(e_{k}\right)_{k \geq 1}$ be the sequence of projections associated to the Markov traces. The following relations hold:

(a) $e_{k} e_{l}=e_{l} e_{k}$ if $|k-l| \geq 2$;

(b) $e_{k} e_{k \pm 1} e_{k}=\beta^{-1} e_{k}$ for every $k$ (see [5]).

Set for $n \geq 0$ and for $k \geq 1$

$$
\begin{aligned}
g_{n}^{k}= & \left(e_{n+k} e_{n+k-1} \cdots e_{k}\right)\left(e_{n+k+1} \cdots e_{k+1}\right) \cdots\left(e_{2 n+k} \cdots e_{n+k}\right) \\
& \in M_{2 n+k+1}, \\
f_{n+1}^{k}= & \gamma_{n} g_{n}^{k}, \quad \text { where } \gamma_{n}=\beta^{n(n+1) / 2}, \text { and } f_{n+1}=f_{n+1}^{1} .
\end{aligned}
$$

The proof of the following lemma is exactly the same as in the case of factors [7], $\S 2$ :

LEMMA 5.9. With the notations above, one has:

(i) $\left(g_{n}^{1}\right)^{*}=g_{n}^{1}=\left(e_{n+1} \cdots e_{2 n+1}\right) g_{n-1}^{1}\left(e_{2 n} \cdots e_{n+1}\right)$;

(ii) $f_{n+1}$ is a projection in $N^{\prime} \cap M_{2 n+2}$;

(iii) $E_{M_{2 n+k}}\left(g_{n}^{k}\right)=\beta^{-(n+1)} g_{n-1}^{k+1}$;

(iv) $E_{M_{n+1}}\left(f_{n+1}\right)=\beta^{-(n+1)}$. 
THEOREM 5.10. Let $N \subset M$ be as above. Denote by $e_{N, n}$ the projection of $L^{2}\left(M_{n}\right)$ onto the closed subspace $\left[N \Omega_{n}\right]$, where $\Omega_{n}$ is the positive vector associated to $\operatorname{tr}_{M_{n}}$. Then there exists an isomorphism $\pi_{n}$ from $\left\langle M, e_{N, n}\right\rangle$ onto $M_{2 n}$ such that

$$
\pi_{n}(x)=x \text { for } x \text { in } M_{n} \text { and } \pi_{n}\left(e_{N, n}\right)=f_{n} .
$$

Proof. By Lemma 5.8, assume that we have for some $n \geq 1$ :

(i) the central support of $f_{n}$ in $M_{2 n}$ equals 1 ;

(ii) $f_{n} x f_{n}=E_{N, n}(x) f_{n}$ for $x$ in $M_{n}$;

(iii) $E_{M_{n}}\left(f_{n}\right)=\beta^{-n}$;

(iv) $M_{2 n}^{n}=\left(M_{n} \cup\left\{f_{n}\right\}\right)^{\prime \prime}$.

Remark that (i) to (iv) hold for $n=1$. Let us check them for $n+1$. Condition (iii) is statement (iv) of Lemma 5.9.

(i) Every central projection of $M_{2 n+2}$ is of the form JqJ where $q$ is a central projection of $M_{2 n}$ and where $J$ is the canonical involution on $L^{2}\left(M_{2 n+1}\right)$. We prove that $g_{n}^{1} J q J \neq 0$ if $q \neq 0$ :

$$
\begin{aligned}
g_{n}^{1} J q J & =\left(e_{n+1} \cdots e_{2 n}\right) e_{2 n+1} g_{n-1}^{1}\left(e_{2 n} \cdots e_{n+1}\right) J q J \\
& =\left(e_{n+1} \cdots e_{2 n}\right) e_{2 n+1} q g_{n-1}^{1}\left(e_{2 n} \cdots e_{n+1}\right) ;
\end{aligned}
$$

hence

by induction hypothesis.

$$
\operatorname{tr}_{M_{2 n+2}}\left(g_{n}^{1} J q J\right)=\beta^{-(n+1)} \operatorname{tr}_{M_{2 n}}\left(q g_{n-1}^{1}\right)>0
$$

(ii) It suffices to show that $f_{n+1} x e_{n} y f_{n+1}=E_{N, n+1}\left(x e_{n} y\right) f_{n+1}$ for $x$ and $y$ in $M_{n}$. As $e_{n+1}$ commutes with $M_{n}$ and as $e_{2 n+1}$ commutes with $f_{n}$, we have:

$$
\begin{aligned}
f_{n+1} x e_{n} y f_{n+1}=\gamma_{n}^{2}\left(g_{n}^{1}\right)^{*} x e_{n} y g_{n}^{1} \\
\quad=\gamma_{n}^{2}\left(e_{n+1} \cdots e_{2 n}\right) g_{n-1}^{1} x\left(e_{2 n+1} \cdots e_{n} \cdots e_{2 n+1}\right) y g_{n-1}^{1}\left(e_{2 n} \cdots e_{n+1}\right) \\
=\left(\gamma_{n} \gamma_{n-1}^{-1}\right)^{2} \beta^{-n-1}\left(e_{n+1} \cdots e_{2 n+1}\right) f_{n} x y f_{n}\left(e_{2 n} \cdots e_{n+1}\right) \\
\quad=\beta^{-1} E_{N, n}(x y) f_{n+1}=E_{N, n+1}\left(x e_{n} y\right) f_{n+1} .
\end{aligned}
$$

(iv) By Kaplansky's density theorem and by induction, it is easy to see that the subspace $M_{2 n-1} e_{2 n-1} e_{2 n} e_{2 n+1} M_{2 n} f_{n} M_{2 n}$ is strongly dense in $M_{2 n+2}$. As $\operatorname{tr}_{M_{2 n}}$ is a Markov trace, and as $M_{2 n}$ is isomorphic to $\left\langle M_{n}, e_{N, n}\right\rangle$, then $M_{2 n} f_{n}=M_{n} f_{n}$ by Lemma 1.2 of [6]. Then by going on in the same way, we see that the subspace

$$
M_{n+1}\left(e_{n+1} \cdots e_{2 n+1}\right) f_{n}\left(e_{2 n} \cdots\right) M_{n+1}
$$

is strongly dense in $M_{2 n+2}$. As $\left(e_{n+1} \cdots e_{2 n+1}\right) f_{n}\left(e_{2 n} \cdots e_{n+1}\right)$ is proportional to $f_{n+1}$, we have that $M_{n+1} f_{n+1} M_{n+1}$ is strongly dense in $M_{2 n+2}$. 
Finally, let us mention the following observation which may be compared to the original definition of index in the case of factors [5]:

Proposition 5.11. Assume that $N \subset M$ is a pair as in Theorem 5.10. Denote by $\operatorname{tr}_{N}$ the restriction to $N$ of the Markov trace $\operatorname{tr}_{M}$. Then the trace $\operatorname{tr}_{N^{\prime}}$ on $N^{\prime} \subset B\left(L^{2}(M)\right)$ defined by

$$
\operatorname{tr}_{N^{\prime}}\left(y^{\prime}\right)=[M: N]^{-1} \operatorname{tr}_{M}\left(c_{N} y^{\prime h_{N^{\prime}}}\right) \text { for } y^{\prime} \text { in } N^{\prime},
$$

is a normalized Markov trace of modulus $[M: N]=\left[N^{\prime}: M^{\prime}\right]$ for the pair $M^{\prime} \subset N^{\prime}$. Moreover one has for every $\xi$ in $L^{2}(M)$ :

$$
[M: N] \operatorname{tr}_{N^{\prime}}\left(e_{\xi}^{(N)}\right)=\operatorname{tr}_{N}\left(e_{\xi}^{\left(N^{\prime}\right)}\right) .
$$

Proof. We have: $D_{M^{\prime}}^{N^{\prime}}(w)=\left(\left(c_{N} w\right)^{\natural_{M}}\right)^{\natural_{N^{\prime}}}$ for $w$ in $Z(N)$. Hence

$$
\begin{aligned}
\operatorname{tr}_{N^{\prime}} \circ D_{M^{\prime}}^{N^{\prime}}(w) & =[M: N]^{-1} \operatorname{tr}_{M}\left(\left(c_{N}\left(\left(c_{N} w\right)^{\natural_{M}}\right)^{\natural_{N^{\prime}}}\right)^{\natural_{M}}\right) \\
& =[M: N]^{-1} \operatorname{tr}_{M}\left(D_{N}^{M}\left(\left(c_{N} w\right)^{\natural_{M}}\right)\right) \\
& =[M: N][M: N]^{-1} \operatorname{tr}_{M}\left(c_{N} w\right) \\
& =[M: N] \operatorname{tr}_{N^{\prime}}(w) \text { for } w \text { in } Z(N) .
\end{aligned}
$$

Moreover, $\operatorname{tr}_{N^{\prime}}(1)=[M: N]^{-1} \operatorname{tr}_{M}\left(c_{N}^{\natural_{M}}\right)=[M: N]^{-1} \operatorname{tr}_{M}\left(D_{N}^{M}(1)\right)=$ 1. Finally, if $\xi \in L^{2}(M)$, we get by definition of $c_{N}=c_{N}\left(L^{2}(M)\right)$ :

$$
\begin{gathered}
\left(e_{\xi}^{\left(N^{\prime}\right)}\right)^{\natural_{N}}=c_{N}\left(e_{\xi}^{(N)}\right)^{\natural_{N^{\prime}}}, \quad \text { hence } \\
{[M: N] \operatorname{tr}_{N^{\prime}}\left(e_{\xi}^{(N)}\right)=\operatorname{tr}_{M}\left(c_{N}\left(e_{\xi}^{(N)}\right)^{\natural^{\prime}}\right)=\operatorname{tr}_{N}\left(e_{\xi}^{\left(N^{\prime}\right)}\right) .}
\end{gathered}
$$

\section{REFERENCES}

[1] A. Connes, On the spatial theory of von Neumann algebras, J. Funct. Anal., 35 (1980), 153-164.

[2] J. Dixmier, von Neumann Algebras, North-Holland, Amsterdam (1981).

[3] F. Goodman, P. de la Harpe and V. Jones, Coxeter-Dynkin diagrams and towers of algebras, Chapters 1, 2, 3, preprint (1986 \& 1987).

[4] U. Haagerup, The standard form of von Neumann algebras, Math. Scand., 37 (1975), 271-283.

[5] V. F. R. Jones, Index for subfactors, Invent. Math., 72 (1983), 1-25.

[6] M. Pimsner and S. Popa, Entropy and index for subfactors, Ann. Scient. Ec. Norm. Sup., 4ème série, 19 (1986), 57-106.

[7] Iterating the Basic Construction, preprint, INCREST, (1986).

[8] S. Sakai, $C^{*}$-Algebras and $W^{*}$-Algebras, Springer, New York (1971). 
[9] H. Schaefer, Topological Vector Spaces, Springer, New York (1980).

[10] M. Takesaki, Theory of Operator Algebras I, Springer, New York (1979).

Received November 20, 1988 and in revised form March 30, 1989. Work supported by the Swiss National Foundation for Scientific Research while the author was visiting UC Berkeley.

UNIVERSITE DE GENEVE

Section de Mathématiques

Case Postale 240

2-4, RUE DE LIËVRE

CH-1211 Geneve 24, SwitzerLand 


\section{PACIFIC JOURNAL OF MATHEMATICS EDITORS}

\author{
V. S. VARADARAJAN \\ (Managing Editor) \\ University of California \\ Los Angeles, CA 90024-1555-05 \\ Herbert Clemens \\ University of Utah \\ Salt Lake City, UT 84112 \\ Thomas ENRIGHT \\ University of California, San Diego \\ La Jolla, CA 92093
}

\section{R. FINN}

Stanford University

Stanford, CA 94305

Hermann FlaschKa

University of Arizona

Tucson, AZ 85721

VAUGHAN F. R. Jones

University of California

Berkeley, CA 94720

STEVEN KeRCKHOFF

Stanford University

Stanford, CA 94305
C. C. MOORE

University of California

Berkeley, CA 94720

Martin ScharlemanN

University of California

Santa Barbara, CA 93106

HAROLd STARK

University of California, San Diego

La Jolla, CA 92093

\section{ASSOCIATE EDITORS}
R. ARENS
E. F. BECKENBACH
B. H. NeumanN
F. Wolf
K. YoshidA (1906-1982)
(1904-1989)

\section{SUPPORTING INSTITUTIONS}
UNIVERSITY OF ARIZONA
UNIVERSITY OF BRITISH COLUMBIA
CALIFORNIA INSTITUTE OF TECHNOLOGY
UNIVERSITY OF CALIFORNIA
MONTANA STATE UNIVERSITY
UNIVERSITY OF NEVADA, RENO
NEW MEXICO STATE UNIVERSITY
OREGON STATE UNIVERSITY
UNIVERSITY OF OREGON
UNIVERSITY OF SOUTHERN CALIFORNIA
STANFORD UNIVERSITY
UNIVERSITY OF HAWAII
UNIVERSITY OF TOKYO
UNIVERSITY OF UTAH
WASHINGTON STATE UNIVERSITY
UNIVERSITY OF WASHINGTON 


\section{Pacific Journal of Mathematics}

Vol. 146, No. $1 \quad$ November, 1990

Primo Brandi and Anna Salvadori, A quasi-additivity type condition and

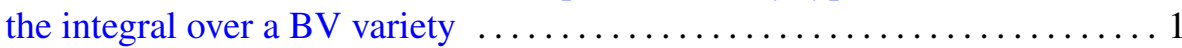

Dong M. Chung, Chull Park and David Lee Skoug, Operator-valued

Feynman integrals via conditional Feynman integrals ..............21

Paul Jolissaint, Index for pairs of finite von Neumann algebras . .........43

Miodrag Mateljević and Miroslav Pavlović, Multipliers of $H^{p}$ and

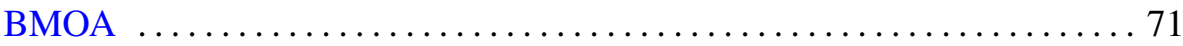

Himadri Kumar Mukerjee, Poincaré cobordism exact sequences and

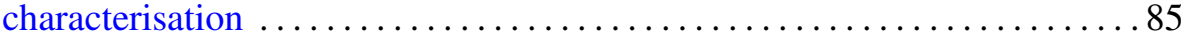

Thomas H. Otway, The coupled Yang-Mills-Dirac equations for differential

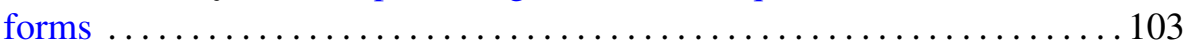

Sechiko Takahashi, Nevanlinna parametrizations for the extended

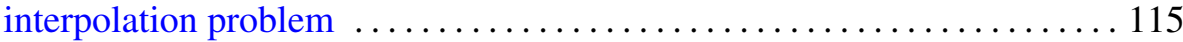

P. C. Trombi, Uniform asymptotics for real reductive Lie groups ........ 131 